\title{
Prospects for a vaccine against HIV
}

Gordon Ada

Early optimism that a vaccine to prevent HIV infection would shortly be forthcoming had waned by the time of the fourth annual international conference on AIDS last year. On the eve of the fifth conference, are prospects any brighter?

VACCINATION is a form of immunoprophylaxis in which all or part of an infectious agent is administered once or a few times to generate an immune response that protects against subsequent infection. Many vaccines to prevent or control viral infections are highly successful. The widespread application of those composed of live (infectious), attenuated (non-pathogenic but immunogenic) virus, such as measles, mumps and rubella and polio viruses have reduced these diseases to minor public-health problems, at least in developed countries. Several attenuated viral vaccines under development, such as rotavirus, also show great promise. Some inactivated virus (rabies, polio) vaccines and a subunit (hepatitis B surface antigen) vaccine are fairly successful.

In view of this impressive record, it seems surprising that all attempts to make a practical vaccine against the human immunodeficiency virus, HIV, have so far been unsuccessful. A successful vaccine is the most direct way of showing that it is possible to stimulate an immune response in humans that will prevent or clear an HIV infection and thus prevent AIDS. The history of epidemics shows that although the mortality rate in an infected population may be very high (for AIDS patients in the United States, it is now more than 50 per cent), some people should unaided overcome the infection and survive. One finding is encouraging. In a cohort of $1,000 \mathrm{HIV}$-seropositive people in Baltimore that have been studied for some time, three reverted and have been seronegative for about 30 months'. This suggests that the active infection has been cleared in these people so that they are now immune. It is also possible that an attenuated form of HIV has evolved. Should HIV be experimentally attenuated for use as a vaccine?

Several properties of HIV complicate the development of effective and safe vaccines ${ }^{2}$. HIV is a retrovirus, which means that its RNA genome is copied to form complementary DNA, some of which integrates with the cellular DNA. Even though the cell may not initially produce infectious virus, it is thought to remain infected indefinitely (a latent infection), and possibly may be activated later to produce virus that could be subject to mutation. Naturally occurring superinfection with another retrovirus might allow recombination to occur and a more virulent strain to be produced. Most people agree that this could happen if attenuated virus was used as a vaccine, so this otherwise attractive option has been shelved. This property of HIV also stresses the need for a vaccine which would prevent infection and hence stop the development of a latent infection.

Another approach, the use of inactivated HIV as a vaccine, is being tried but is not widely favoured because there is a poor record of such vaccines against retroviruses. This type of immunization often fails to induce the production of cytotoxic $\mathrm{T}$ lymphocytes, cells which kill virusinfected cells as part of the normal

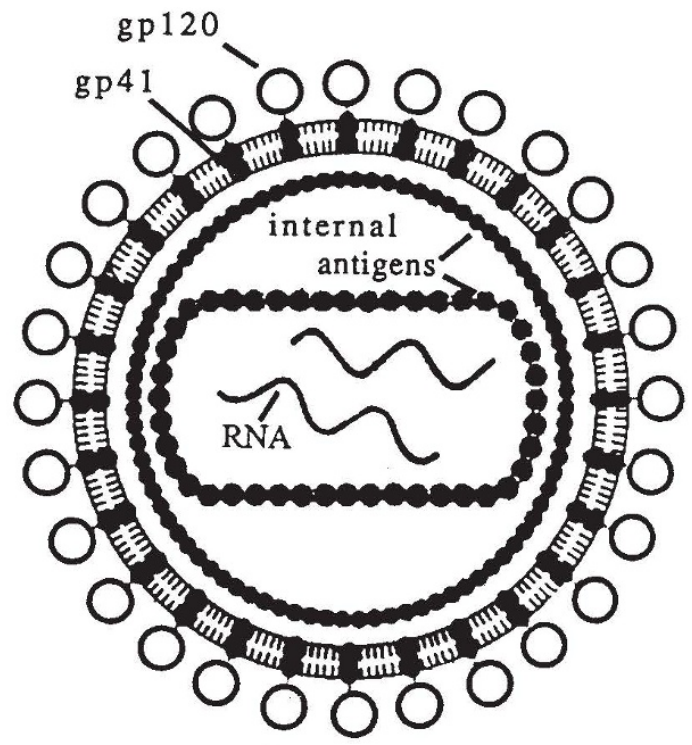

Some features of HIV. The Env protein, gp160, has two components, gp120 and gp41, the latter projecting on both sides of the lipid membrane.

immune response. Furthermore, growing large amounts of HIV may be impractical.

Perhaps encouraged by the success of the hepatitis $\mathrm{B}$ vaccine, most effort is now directed to the development of a safe and effective subunit vaccine against HIV based on the surface envelope antigen gp160, which is composed of gp120 and gp41 (see figure). To judge from many other viral infections, the vaccine should induce antibodies that neutralize viral infectivity as well as cytotoxic T lymphocytes. Both responses may develop, although often late during the natural infection. Much effort has been made to analyse in detail the properties of gp 160 , with both encouraging and disturbing results.
One particularly disturbing finding is that there is a great amount of antigenic variation in gp 160 - at least 25 per cent of the amino acids may change $e^{3.4}$. A range of antigenically distinct HIVs has been isolated from one infected individual ${ }^{5,6}$. In the case of other viruses, marked antigenic drift in the protective antigen(s) of a virus has been a barrier to the development of effective vaccines (influenza, rhinoviruses). In HIV, one such segment occurs as a disulphide-bridged loop containing 18 amino acids, most of which are variable. In animal experiments, it has been found that this loop is the immunodominant site recognized by antibodies which neutralize the virus and thus prevent infection. One hopeful sign is that there seems to be a pattern of variation $^{5.6}$; if so, a few sequences might give a broadly protective response. In sharp contrast, there is some experimental evidence that a conserved (non-variable) amino-acid sequence, present on a part of gp 160 in the virus particle beyond but near the inner surface of the viral lipid membrane, is recognized by neutralizing antibody from infected people ${ }^{7.8}$, a finding that may be unique. It is encouraging that the less sensitive assays used previously for assaying viral infectivity have been replaced by quantitative techniques, allowing surer detection of important target sequences (called epitopes) ${ }^{9}$.

An antibody against gp120 may aiso have other functions. One useful role is to facilitate cell-mediated lysis of already infected cells (antibodydependent cellular cytotoxicity). It has been reported that seropostive patients who have not progressed to disease may have high titres of this antibody. Antibody may also accelerate the uptake of virus into cells such as macrophages and monocytes that have receptors recognizing antibody/virus/complement complexes and where some viruses are destroyed. However, HIVs productively infect these cells and this may be the way the virus is transported to different parts of the body, including immunoprivileged sites such as brain, epididymis and possibly bone marrow, which then become sanctuaries where infectious virus is continually produced.

Progress has been made in identifying epitopes recognized by both helper and cytotoxic $\mathrm{T}$ lymphocytes. At least one 
epitope is in a variable region of gp160. Findings from many other systems, such as malaria, indicate that a vaccine may need to contain several different T-cell epitopes to generate adequate immune responses in most recipients in an outbred population. Therefore, T-lymphocyte epitopes present in other viral antigens will need to be identified and incorporated into a vaccine. Several such epitopes have recently been described

Two other properties of gp160 are of interest. The first is that there are similarities between several amino-acid sequences in the Env antigen and in some normal

Retention/deletion of biological active segments of HIV antigens in the construction of a 'synthetic' vaccine

\section{Retain}

Epitopes recognized by: neutralizing antibodies (gp160); antibodies involved in lysis of infected cells - ADCC (gp160); helper and cytotoxic T lymphocytes (gp160 and other viral antigens)

Peptide segments involved in binding to cellular receptors and in fusion to the cell membrane (gp160).

\section{Delete}

Epitopes recognized by antibodies which enhance infection of cells (macrophages, monocytes) (gp160)

Sequences showing homology with norma self components (gp160)

Sequences inducing suppression of immune responses (gp160)*

*Alternatively, it might be advantageous to include this sequence in the vaccine and generate a strong antibody response to $\mathrm{it}^{7}$.

host proteins. One of the latter is a class II HLA molecule, present at the surface of many host cells. Such findings raise concern that immunization with intact gp160 induces autoimmunity, possibly leading to autoimmune disease. The second finding is that a synthetic peptide representing a sequence in gp160 (in the transmembrane component gp41 - see figure) inhibits lymphocyte proliferation ${ }^{10}$. In HIV-seropositive individuals, the presence of antibody against this immunosuppressive peptide is reported to be associated with absence of disease". This result may explain earlier reports that infected people have suppressor-cell activity which delays or prevents a protective antibody response.

A form of immunotherapy of considerable interest has the aim of inhibiting the interaction between the virus and CD4, the cellular receptor of HIV. One approach is to block the receptor itself, but as this molecule is involved in T-lymphocyte activation, this has the potential for undesirable consequences. Alternatively, 'soluble' CD4 molecules (or their equivalent) might be provided in sufficient amounts to bind to most if not all receptor-binding sites on the virus and so prevent infection of susceptible cells. Daily injections of CD4 to monkeys infected with a simian immunodeficiency virus (SIV) related to HIV have recently been shown to have beneficial effects ${ }^{12}$ Methods have now been described ${ }^{1.3}$ allowing such a reagent to be modified and remain in the circulation for a long time, thus requiring fewer administrations (which must be a requirement if this approach is to be feasible). As both CD4 and the receptor-binding site of gp 160 are conserved, this approach bypasses the difficulties raised by antigenic variation. Specific antibodies (anti-idiotypes) mimicking the receptor molecule are also being investigated.

How can all this information best be used for the development of a vaccine? It is now possible to dissect a molecule to retain desirable and delete unwanted segments, or to modify selected features of it. The table indicates the activities that might be retained or deleted in the final antigen preparation. Furthermore, different presentation mechanisms could be used. Cytotoxic $\mathrm{T}$ lymphocyte responses might best be generated using a recombinant live vector (vaccinia, adeno, polio viruses), whereas administration with particular adjuvants, such as immunostimulatory complexes or liposomes, might give the best antibody responses ${ }^{14}$.

There is a great restriction on some HIV studies because of the lack of appropriate animal models. The recent description of an immunodeficient mouse strain which can be transplanted with human cells and infected with HIV offers the hope that susceptible animals will become available in sufficiently large numbers to test, at an early stage, different approaches to vaccine development.

There is one area that has received little attention. HIV is transmitted in four main ways - sexual intercourse, needle sticking, from mother to child and blood transfusion. All may involve free and/or cell-associated virus, and there is reason to believe that the latter may be an important mechanism. It is only recently that attention has been paid to defining the levels of free and cell-associated virus in semen. Not only is there great variation in cell numbers, in the degree of inflammation and in the amount of free or cellassociated virus, but semen also contains immunosuppressive factors ${ }^{15}$. The information required to develop the best vaccine will not be available until the state of virus in infected cells is characterized and the mechanism by which the virus infects the recipient is known.

Candidate vaccines are being developed and produced in the industrialized countries, where the responsibility of testing the product for safety and immunogenicity is generally accepted. For social and legal reasons there have been difficulties in the recruitment of volunteers, but several phase 1 trials of putative vaccines are now under way. It seems probable that later trials (phase 2 and 3) will be carried out in developing countries. Furthermore, as vaccination is likely to be the only practical way to prevent and a major means for the control of HIV infection in the developing countries, it is critical that proper guiding principles are followed: beneficence (the obligation to do good rather than harm); justice (equal sharing among the population of the risks and of the subsequent benefits); and autonomy (subjects have freedom of choice about participating in research activities) ${ }^{16}$.

The great increase in knowledge about the virus, particularly the envelope protein, during the past year means that there are now more leads to follow and hence better prospects for effective, safe HIV vaccines. But there are still substantial obstacles. The Env antigen exists as a trimeric molecule and is enveloped in a 'shroud' of sugar molecules so that some peptide sequences are 'immunosilent'. An immune response to these may be useful. Some neutralizing antibodies recognize discontinuous epitopes". If these are important, their structure needs to be determined. Many of the early successful vaccines were developed without much understanding about the properties of the virus or about how the immune responses required for protection were generated. Viruses such as HIV present a more formidable challenge.

Fortunately, there is always the prospect of an unexpected or novel result. The encouraging findings with a chimaeric poliovirus (see the paper by Almond and collaborators on page 385 of this issue ${ }^{17}$ ) containing a conserved epitope of gp160, which both reacts well with and generates human HIV-neutralizing antibody ${ }^{7.8}$, raises our hopes. Perhaps this is one vulnerable spot in HIV.

Gordon Ada is in the Department of Immunology and Infectious Diseases, Hygiene and Public Health, Johns Hopkins University, Baltimore, Maryland 21205, USA.

Farzadegan, H et al Ann Int Med 108,785-790 (1988)

Add G L AIDS 1, 295-303(1988)

. Modrow, S. et al. J. Virol. 61. 570-578 (1987).

4. Starcich, B.R. et al. Cell 45, 637-648 (1986).

5. Saag, M.S. et al. Nature 334, 440-444 (1988)

Fisher. A.G. et al, Nature 334, 444-447 (1988)

Chanh. T.C et al EMBO J 5, 3065-3071 (1986)

Dalgleish, A.G. et al. Virology 165, 209-215 (1988)

WHO Workshop on the Measurement and Significance of Neutralizing Antibody to HIV and SIV. WHO Bull. (submitted).

10. Ruegg, C.L., Monell, C.R. \& Strand, M. J. Virol. (in the press)

1. Klasse, P.J., Pipkorn, R. \& Blomberg, J. Proc. natn. Acad Sci. U.S.A. 85, 5225-5229(1988).

12. Watanabe, M. et al. Nature 337, 267-270 (1989)

13. Capon, D.J.et al. Nature 337, 525-531 (1989)

14. Ada, G.L. in AIDS and the New Viruses (eds Dalgleish. A.G. \& Weiss. R.) (Academic. London, In the press)

15. Anderson, D.J. et al. in The Heterosexual Transmission of AIDS (ed. Alexander, N.) (Liss. New York, in the press)

16. World Health Organization. Consultation: Criteria for International Testing of Candidate HIV Vaccines (WHO. Geneva, 1989)

17. Evans. D.J. et al. Nature 339, 385-388 (1989) 